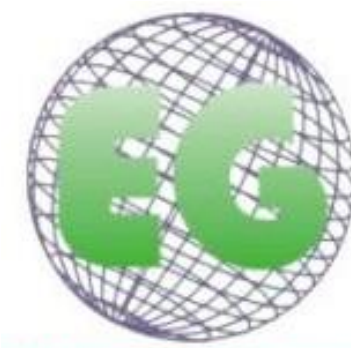

ISSN 1695-6141 $N^{\circ} 50$

\title{
Burnout y engagement en estudiantes universitarios de enfermería
}

Burnout and engagement among university nursing students

\author{
Cristina Liébana-Presa ${ }^{1}$ \\ $M^{\text {a }}$ Elena Fernández-Martínez ${ }^{2}$ \\ Ana $\mathrm{M}^{\mathrm{a}}$ Vázquez-Casares ${ }^{1}$ \\ Ana Isabel López-Alonso ${ }^{2}$ \\ $\mathrm{M}^{\mathrm{a}}$ Aurora Rodríguez-Borrego ${ }^{3}$
}

1 Profesor Colaborador fijo. Departamento de Enfermería y Fisioterapia. Grupo de investigación Cuidados para la Salud (CUIDAS). Universidad de León. Ponferrada, León. España.

${ }^{2}$ Profesora Titular de Universidad. Departamento de Enfermería y Fisioterapia. Grupo de investigación cuidados para la salud (CUIDAS). Universidad de León. Ponferrada, León. España.

${ }_{3}$ Profesora Titular de Universidad. Departamento de Enfermería. Universidad de Córdoba/ Hospital Universitario Reina Sofía/ Instituto Maimónides de Investigación Biomédica de Córdoba (IMIBC). Grupo de investigación IMIBIC-GA2. Cuidados Enfermeros Integrales perspectiva Multidisciplinar. Córdoba. España.

\section{E-mail: cliep@unileon.es}

http://dx.doi.org/10.6018/eglobal.17.2.268831

Recibido: 26/09/2016

Aceptado: 20/11/2016

\section{RESUMEN:}

Los universitarios se encuentran sometidos a cargas académicas prolongadas que ocuparan la mayor parte de su tiempo. La capacidad para hacer frente a estas situaciones de estrés durante su formación, dependerá ente otras cosas, de la disposición personal para afrontar las demandas académicas.

Objetivo: Describir el nivel de burnout (agotamiento, cinismo y eficacia académica) y el compromiso académico a través de la variable engagement (absorción, dedicación y vigor), además de analizar las asociaciones, relaciones y predicciones entre estas variables en estudiantes de Enfermería.

Material y método. Estudio descriptivo transversal en 1009 sujetos. Instrumentos de recogida de datos: Maslach Burnout Inventory y Utrech Work Engagement en versión estudiantes.

Resultados: Las dimensiones del burnout y engagement correlacionan entre sí. A mayor eficacia académica menos agotamiento y cinismo y mayor puntuación en todas las dimensiones del engagement académico. El vigor y la dedicación predicen puntuaciones más altas en eficacia académica. Las mujeres se perciben con puntuaciones más bajas en cinismo y más altas en eficacia académica, absorción y dedicación.

Conclusiones: Los alumnos muestran más burnout cuando manifiestan altas puntuaciones de agotamiento y cinismo y bajas en eficacia académica, además valores altos en las tres dimensiones del engagement indican alto compromiso con sus estudios. La relación entre estas variables pone de manifiesto la diferencia entre hombres y mujeres y la influencia positiva del engagement en la eficacia académica del estudiante. El fomento de engagement puede ser una oportunidad para superar las tareas académicas y para enfrentarse al mundo laboral con mayor probabilidad de éxito. 
Palabras clave: Engagement; Burnout; Enfermería; Educación en enfermería

\begin{abstract}
:
University students are tasked with a protracted academic workload that occupies the greater part of their time. Their ability to cope with the stress this entails during their degree course will partly depend on their personal response to these academic demands.

Objective: to describe nursing students' level of burnout (exhaustion, cynicism and academic efficacy) and academic engagement through the variable engagement (absorption, dedication and vigour), and to analyse associations, relations and predictions between these variables.

Materials and method: cross-sectional descriptive study with 1009 subjects. Data collection instruments: student versions of the Maslach Burnout Inventory and the Utrecht Work Engagement Scale.

Results: the dimensions of burnout and engagement correlated with each other. The higher the score for academic efficacy, the lower the scores for exhaustion and cynicism and the higher the score in all dimensions of academic engagement. Vigour and dedication predicted higher scores for academic efficacy. Females' self-perceptions indicated lower scores for cynicism and higher scores for academic efficacy, absorption and dedication.

Conclusions: students evidenced more burnout when they obtained high scores for exhaustion and cynicism and low scores for academic efficacy. In addition, high scores for the three dimensions of engagement indicated high commitment to their studies. The relationship between these variables indicates a difference between males and females and a positive influence of engagement on students' academic efficacy. Promoting engagement could help students to deal with academic tasks and enter the labour market with a greater probability of success.
\end{abstract}

Keywords: Engagement; Burnout; Nursing; Nursing Education

\title{
INTRODUCCIÓN
}

Una consecuencia de estrés laboral es el síndrome de burnout y aunque no existe una definición unánimemente aceptada ${ }^{(1,2)}$ parece que hay acuerdo en entender el burnout como un fenómeno psicológico que surge como respuesta a factores estresantes crónicos interpersonales en el trabajo ${ }^{(3)}$, es un síndrome caracterizado por tres dimensiones básicas, agotamiento emocional, despersonalización o cinismo y una falta de realización personal ${ }^{(4)}$. El agotamiento describe una pérdida progresiva de energía en las tareas que se realizan, es la manifestación primaria del burnout y la dimensión que más comúnmente se declara. La despersonalización, se manifiesta por irritabilidad, actitudes negativas y respuestas insensibles hacia las personas. La tercera dimensión del burnout, falta de realización personal, está caracterizada por respuestas negativas hacia sí mismo y hacia las actividades que se desempeñan, las personas expresan falta de productividad en el trabajo e inadecuación a las tareas profesionales $^{(5)}$.

El interés que el término de burnout está teniendo en la actualidad ${ }^{(6)}$ ha dispuesto la extensión de este síndrome a muestras pre-ocupacionales como los estudiantes universitarios, la investigación sobre el burnout académico ${ }^{(7,8)}$ indica que los estudiantes también presentan desgaste por las tareas que realizan.

Un hecho relevante en la investigación sobre burnout ha sido el estudio de un constructo opuesto denominado engagement ${ }^{(3,9)}$. El engagement o compromiso psicológico, coincide con el movimiento de la psicología positiva que trata de potenciar los aspectos favorecedores de la vida humana para construir cualidades positivas y evitar la dominante preocupación por los aspectos negativos de las personas ${ }^{(10)}$. El engagement es definido ${ }^{(9)}$ como un estado positivo de la mente que se caracteriza por energía, implicación y eficacia. Más concretamente, el engagement es entendido como un constructo motivacional positivo relacionado con el trabajo que 
está caracterizado por los atributos de vigor, dedicación y absorción ${ }^{(11)}$. Es un estado cognitivo-afectivo más que un estado específico y temporal, que persiste en el tiempo y que no está focalizado sobre un objeto o una conducta específica ${ }^{(9,12)}$. El vigor hace referencia a los altos niveles de energía con las tareas desarrolladas, así como un fuerte deseo de esfuerzo y persistencia ante la aparición de obstáculos en el camino. La característica de dedicación, responde a altos niveles de significado del trabajo, entusiasmo, inspiración, orgullo y el reto en las tareas realizadas. El factor de absorción implica altos niveles de concentración y de felicidad mientras se realiza una actividad. La persona, se encuentra inmersa en lo que hace y experimenta la sensación de que el tiempo pasa muy deprisa.

Cuando se habla del burnout y engagement como constructos contrarios, algunas investigaciones ${ }^{(3,9,13)}$ matizan que el vigor y la dedicación del engagement son dimensiones opuestas al agotamiento y cinismo del burnout, consideradas éstas últimas dimensiones, el "corazón del burnout ${ }^{(14) "}$.

Los últimos estudios sobre estos constructos muestran un creciente interés por el burnout $^{(15)}$ y engagement en el ámbito académico en muestras de estudiantes universitarios $^{(16-19)}$, tras análisis confirmatorios de la validez y la consistencia interna de los instrumentos que evalúan estas variables ${ }^{(12)}$, también se ha estudiado si el engagement añade valor explicativo en la predicción de indicadores de bienestar ${ }^{(9,20)}$. Además, ambos constructos, se han relacionado con otras variables como la inteligencia emocional ${ }^{(21,22)}$ y la satisfacción ${ }^{(23)}$. En esta misma línea, recientemente, se han analizado la influencia de estos constructos junto a la eficacia y rendimiento académico $^{(24,25)}$, ampliando las perspectivas y entendiendo el burnout como crisis de eficacia o la eficacia como motor de engagement ${ }^{(14,26,27)}$.

En las universidades públicas de Castilla y León (España), contexto educativo de la presente investigación, los estudiantes universitarios y en particular los de enfermería, deben superar los retos académicos programados en los planes de estudio que ocuparán la mayor parte de su tiempo. Los alumnos, se implican en las tareas teóricas organizadas bajo un estricto horario y además deben acudir a las prácticas clínicas, donde se les exige comportamientos y actividades productivas, conocimientos todos ellos, sometidos a evaluación. Las instituciones públicas de educación superior, deben prestar atención al estado emocional de sus estudiantes en situaciones de aprendizaje ${ }^{(27)}$ puesto que la capacidad para hacer frente a las situaciones de estrés durante su educación, dependerá de la disposición personal para afrontar las demandas académicas ${ }^{(28)}$.

Los objetivos del este estudio son describir el nivel de burnout y engagement en los estudiantes de enfermería de las universidades públicas de la región de Castilla y León (España). Analizar la relación, asociación y predicción entre las dimensiones de agotamiento emocional, cinismo, eficacia académica del burnout con los niveles de absorción, dedicación y vigor del engagement. Así como, analizar las diferencias de medias, comparando los grupos de hombres y mujeres. Estos objetivos se presentan para conocer más sobre estas variables y su relación, con el fin de identificar posibles necesidades de intervención que puedan garantizar la calidad de la enseñanza y dotar al universitario de recursos para su futuro académico y profesional, competencias de éxito para la institución universitaria pública. 


\section{MATERIAL Y MÉTODO}

\section{Diseño de investigación}

Investigación cuantitativa sin intervención. Estudio descriptivo transversal. Se analizaron las diferencias entre los grupos de hombres y mujeres así como las correlaciones y predicciones que se establecen entre las distintas variables a estudio, burnout y engagement.

\section{Sujetos y ámbito de estudio}

La población de estudio fueron los estudiantes de la titulación de Enfermería matriculados en el curso académico 2009/2010. Los estudiantes pertenecían a las cuatro Universidades públicas que existen en Castilla y León (España): universidad de Burgos, universidad de León, universidad de Salamanca y universidad de Valladolid.

\section{Instrumentos de recogida de datos}

-Cuestionario Maslasch Burnout Inventory-Students Survey (MBI-SS), adaptado para medir el nivel de burnout en estudiantes universitarios ${ }^{(12)}$ y validado en la población española ${ }^{(28)}$. Esta escala contiene 15 ítems que evalúan las dimensiones de agotamiento $(\square=0,876)$, cinismo $(\square=0,826)$ y eficacia académica $(\square=0,663)$; todos estos aspectos relacionados con sentimientos, creencias y conductas relacionadas con la experiencia como "estudiante" en la universidad. El intervalo de respuesta de los distintos ítems, oscila desde 0 a 6 en una escala tipo Likert en la que 0 significa totalmente en desacuerdo y 6 totalmente de acuerdo. El estudiante está más quemado cuando manifiesta altas puntuaciones en agotamiento y cinismo y bajas puntuaciones en su creencia de eficacia académica.

-Cuestionario Utrech Work engagement Student (UWES-S) adaptado para medir el nivel de engagemnet en estudiantes universitarios ${ }^{(12)}$ y validado en la población española ${ }^{(29,30)}$. Es una escala de 17 ítems que se usa para medir las tres dimensiones de este constructo en estudiantes: vigor $(\square=0,792)$, dedicación $(\square=0,791)$ y absorción $(\square=0,802)$. Las respuestas de los encuestados se cuantifican según una escala tipo Likert, en la que 0 significa totalmente en desacuerdo y 6 totalmente de acuerdo con la afirmación reflejada en el ítem. Altas puntuaciones en estas dimensiones reflejan elevados niveles de engagement académico.

Las características socio-demográficas fueron: sexo, edad, y universidad donde cursaban sus estudios.

\section{Procedimiento}

Se solicitó por escrito a las universidades participantes el permiso para la administración de los cuestionarios. Tras la respuesta favorable por parte de los centros, se concretó con las cuatro universidades públicas el día y la hora más adecuados para el desplazamiento a la Universidad correspondiente y proceder con la recogida de datos. En el contexto del aula y previamente a la administración de los cuestionarios, la investigadora principal informó oralmente y por escrito, a través de la hoja de información y consentimiento informado (que no precisaron firmar, para preservar su anonimato), de los objetivos del estudio y contestó a las preguntas que los estudiantes plantearon al respecto. Tras asegurar a todos los individuos 
participantes el anonimato y el carácter voluntario de su participación en el estudio, se les distribuyó los cuestionarios para que los cumplimentasen de forma individual. El tiempo empleado en cumplimentar los cuestionarios fue de aproximadamente 20 minutos.

\section{Análisis de los datos}

Las técnicas de análisis de datos utilizadas fueron los estadísticos descriptivos (tendencia central y de dispersión), análisis de correlación lineal (coeficiente de correlación de Pearson), análisis T-test con la prueba t de student y el análisis de regresión lineal múltiple, para elaborar predicciones sobre una variable a partir de otras. Con el fin de hallar los indicadores psicométricos de los instrumentos de medida, se analizó el coeficiente de fiabilidad alfa de Cronbach ( $\square$ ).

La base de datos y los análisis estadísticos citados se han realizado mediante el programa informático SPSS 21.0 (Statistical Package for the Social Sciences).

\section{Consideraciones éticas}

El protocolo de estudio y los instrumentos fueron aprobados por la Comisión ejecutiva del Comité de Ética de la Universidad de León.

La investigación propuesta respeta los principios fundamentales de la declaración de Helsinki, así como los principios éticos de la profesión enfermera que sobre investigación tiene establecido el código Deontológico de la Enfermería Española.

Los cuestionarios fueron acompañados de la hoja de información y del consentimiento verbal y escrito.

\section{RESULTADOS}

Participaron de manera voluntaria y anónima, 1009 estudiantes de enfermería matriculados en las cuatro Universidades públicas de Castilla y León (España). La media de edad fue de 21,53 años. En la tabla $1^{(20)}$ se muestran las características de los sujetos y la distribución de los participantes por universidades, el sexo predominante fue el femenino con 859 mujeres $(85,1 \%)$ y 127 hombres $(12,7 \%)$, aquellos estudiantes que no cumplimentaron o respondieron de forma incorrecta a la variable sexo fueron considerados bajo la categoría desconocidos. La cifra de desconocidos ascendió a 23 participantes de la muestra (2,3\%).

Tabla 1 - Características socio-demográficas de los estudiantes de enfermería de las universidades públicas de Castilla y León (España).

\begin{tabular}{lllll}
\hline Universidades públicas & Hombres & Mujeres & Desconocido & Total $\mathrm{N}=1009$ \\
\hline Universidad de León & 52 & 376 & 7 & $435(43,1 \%)$ \\
Universidad de Salamanca & 20 & 100 & 5 & $125(12,4 \%)$ \\
Universidad de Valladolid & 43 & 269 & 9 & $321(31,8 \%)$ \\
Universidad de Burgos & 12 & 114 & 2 & $128(12,7 \%)$ \\
\hline Total & $127(12,6 \%)$ & $859(85,1 \%)$ & $23(2,3 \%)$ & 1009 \\
\hline
\end{tabular}

Las medias, desviaciones típicas, correlaciones de Pearson y coeficientes de fiabilidad de los instrumentos de medida se muestran en la Tabla 2. Como se puede 
observar, el alfa de Cronbach supera o se aproxima en las dimensiones analizadas al criterio de 0,70 recomendado ${ }^{(31)}$.

Tabla 2 - Estadísticos descriptivos y coeficientes de fiabilidad de los instrumentos de medida del burnout y engagement de los estudiantes de enfermería de las universidades públicas de Castilla y León (España).

\begin{tabular}{lllllll}
\hline & 1 & 2 & 3 & 4 & 5 & 6 \\
\hline 1.Agotamiento & 1 & & & & & \\
2. Cinismo & $0,494^{*}$ & 1 & & & & \\
3. Eficacia académica & $-0,071^{* *}$ & $-0,236^{*}$ & 1 & & & \\
& & & & & & \\
4. Absorción & $-0,062$ & $-0,165^{*}$ & $0,457^{*}$ & 1 & & \\
5. Dedicación & $-0,150^{*}$ & $-0,486^{*}$ & $0,434^{*}$ & $0,544^{*}$ & 1 & \\
6. Vigor & $-0,246^{*}$ & $-0,251^{*}$ & $0,530^{*}$ & $0,720^{*}$ & $0,532^{*}$ & \\
Media & 2,6491 & 1,2183 & 4.1199 & 3,2453 & 4,3943 & 3,0739 \\
Desviación típica & 1,29590 & 1,10736 & 0,76800 & 0,98957 & 0,95033 & 0,98416 \\
Alfa de Cronbach & 0,876 & 0,826 & 0,663 & 0,802 & 0,791 & 0,792 \\
\hline
\end{tabular}

* La correlación es significativa al nivel 0,01 (bilateral).

${ }^{* *}$ La correlación es significante al nivel 0,05 (bilateral).

Según se observa en la Tabla 2, los resultados explican que las dimensiones del burnout se correlacionan entre sí, además, la asociación entre agotamiento y cinismo es más fuerte que la hallada para la eficacia académica. La eficacia académica presenta una correlación moderada en positivo con las variables del engagement; los resultados evidencian que cuanto mayor sea la eficacia académica mostrada por los estudiantes, menores son las puntuaciones obtenidas para el agotamiento y cinismo y mayor es el engagement (absorción, dedicación y vigor). Las tres dimensiones del engagement correlacionan entre sí de manera significativa y en sentido positivo.

Para conocer si las variables se comportan de diferente manera en función del sexo de los individuos, se utiliza la prueba t para muestras independientes (ver Tabla 3). Se encontraron diferencias estadísticamente significativas en la comparación de las medias entre mujeres y hombres para las puntuaciones de cinismo, eficacia académica, absorción y dedicación. Las mujeres puntúan significativamente más alto en estas variables que los hombres excepto en el cinismo donde ellas puntúan más bajo.

Tabla 3 - Descriptivos y prueba t en función del sexo de los estudiantes de enfermería de las universidades públicas de Castilla y León (España).

\begin{tabular}{llllll}
\hline & Sexo & Media & Desviación típica & $\mathrm{t}$ & $\mathrm{p}$ \\
\hline Cinismo & Mujer & 1,1554 & 1,06164 & 4,421 & 0,000 \\
& Hombre & 1,6142 & 1,26724 & & \\
Eficacia académica & Mujer & 4,1477 & 0,76564 & 2,712 & 0,007 \\
& Hombre & 3,9499 & 0,77174 & & \\
Absorción & Mujer & 3,2724 & 0,982136 & 2,088 & 0,037 \\
& Hombre & 3,0749 & 1,04499 & & \\
Dedicación & Mujer & 4,4241 & 0,93924 & 2,637 & 0,009 \\
& Hombre & 4,1841 & 1,04111 & & \\
\hline
\end{tabular}


Tras la evidencia de correlaciones entre la eficacia académica y el resto de las variables estudiadas, el análisis de regresión lineal múltiple muestra que el modelo 2 explica el $31,1 \%$ de la varianza de la eficacia académica, tal y como se muestra en la Tabla 4. Para este análisis, se ha seguido el modelo por pasos (criterio: Prob. de F para entrar $<=0,050$, Prob. de $F$ para salir $>=0,100$ ). Las variables vigor (beta $=0,417$, $\mathrm{t}=-13,335$ y $\mathrm{p}<0,001$ ) y dedicación (beta=0,212, $\mathrm{t}=6,776$ y $\mathrm{p}<0,001$ ) son las que explican más variabilidad. El aumento en la puntuación de vigor y dedicación (engagement), percibido por los estudiantes, predicen puntuaciones más altas en eficacia académica.

Tabla 4. Regresión lineal para la variable dependiente eficacia académica, estudiantes de enfermería de las universidades públicas de Castilla y León (España).

\begin{tabular}{lll}
\hline Variables predictoras & Modelo 1 Beta & Modelo 2 Beta \\
\hline Vigor & 0,530 & $0,417^{*}$ \\
Dedicación & & $0,212^{*}$ \\
$\mathrm{R}$ & 0,530 & 0,559 \\
$\mathrm{R}^{2}$ & 0,280 & 0,313 \\
$\mathrm{R}^{2}$ corregida & 0,280 & 0,311 \\
${ }^{*} \mathrm{p}=0,000$ & &
\end{tabular}

\section{DISCUSIÓN}

Los resultados obtenidos muestran que todas las dimensiones del engagement están fuertemente correlacionadas entre sí, en sentido positivo, en los estudiantes de Enfermería de Castilla y León (España). La dedicación es la variable del engagement que ha obtenido valores más elevados, este dato se confirma con otros estudios ${ }^{(16,23)}$ donde además se han encontrado relaciones entre esta variable con satisfacción, gestión del tiempo o el bienestar en estudiantes ${ }^{(20)}$. En el caso del burnout, la asociación es positiva y moderada para el agotamiento y cinismo, y negativa y más débil en el caso de la eficacia académica y los otros dos factores del constructo. Estos datos fortalecen el carácter consistente de las dos dimensiones del corazón del burnout y la tendencia a analizar el burnout como crisis de auto-eficacia ${ }^{(18)}$, de manera que cuanto mayor sea la eficacia académica mostrada por los estudiantes de Enfermería de las universidades públicas de Castilla y León, menores serán sus niveles de agotamiento y cinismo. Como se esperaba, se confirman otros resultados obtenidos en esta línea que han encontrado asociaciones muy aproximadas a las halladas en el presente estudio ${ }^{(9,14,17,19,21,26,27)}$.

Asimismo, las investigaciones que tratan de identificar las diferencias en función del sexo de los sujetos, muestran que no hay unanimidad, así en algunos estudios ${ }^{(21)}$ no han encontrado diferencias estadísticamente significativas en ninguna de las tres dimensiones del burnout aunque sí hallaron valores significativamente más elevados de dedicación para las mujeres. Sin embargo, en otras investigaciones ${ }^{(17)}$, no se encontraron diferencias de medias en función del sexo para estas variables estudiadas. En la población del estudio que se está presentado, al igual que en el estudio realizado por otros autores ${ }^{(1)}$ se han encontrado diferencias significativas en función del sexo para el cinismo, eficacia, absorción y dedicación. Las mujeres adoptan menos actitudes negativas y distantes, se muestran menos cínicas y se valoran con mayor eficacia, además presentan niveles más elevados de absorción y entusiasmo, inspiración, orgullo y desafío (dedicación) que los hombres. 
Respecto a las relaciones entre burnout y engagement, los resultados obtenidos se apoyan en otras investigaciones empíricas ${ }^{(9,12,14,17,26)}$. Así, las conclusiones de investigaciones con estudiantes de turismo ${ }^{(13)}$ sugieren que la naturaleza desfavorable de burnout (altos niveles de agotamiento y cinismo y poca eficacia) disminuye el compromiso de los estudiantes con su actividad académica (vigor, dedicación y absorción académica disminuida). Al contrario, otros resultados ${ }^{(24)}$ en una muestra de primero de estudiantes de medicina analiza cómo éstos se encuentran comprometidos y enfocados académicamente (altos niveles de engagement); mientras que en relación a su opuesto, burnout, los alumnos presentaban un bajo grado de agotamiento o cansancio emocional. En la muestra estudiada se observa que el factor eficacia académica correlaciona significativamente en positivo con el engagement y en negativo con el burnout, es decir, los alumnos con elevadas expectativas personales y auto-evaluación positiva, también presentan menos sentimientos de desgaste o agotamiento, menor cantidad de adopción de actitudes negativas o cinismo, así como una mayor motivación para las tareas caracterizada por vigor, dedicación y absorción. El análisis de regresión muestra la influencia de las variables vigor y dedicación sobre la eficacia académica, así los futuros profesionales de enfermería estudiados, con altos valores en vigor y dedicación puntúan alto en eficacia académica.

\section{Limitaciones}

Los resultados obtenidos no permiten concluir acerca de la causalidad existente entre las variables estudiadas, sería para ello necesario desarrollar estudios longitudinales o de carácter experimental que logren profundizar en esta cuestión.

Los sujetos estudiados no son representativos de la población universitaria, ya que solamente provienen la rama de ciencias de la salud, enfermería.

Existe también un sesgo en cuanto al sexo, ya que el conjunto de sujetos no es homogéneo para a esta variable, por lo que los resultados y conclusiones sobre las diferencias de género deben ser tomados con cautela.

Finalmente, este estudio ha utilizado una medida de auto-informe para la recopilación de datos donde los resultados podrían haber sido exagerados

\section{CONCLUSIONES}

Los alumnos de enfermería de Castilla y León (España) se muestran más quemados cuando manifiestan altas puntuaciones de agotamiento y cinismo y bajas en autoeficacia. Además los resultados relativos a las tres dimensiones del engagement indican que están altamente correlacionadas entre sí, por lo que altas puntuaciones en estas escalas indican niveles elevados de engagement académico.

Las mujeres universitarias que participaron en el estudio, se muestran menos cínicas y se valoran con mayor eficacia académica. Además, presentan niveles más elevados de absorción y dedicación que los hombres.

Los alumnos con elevada eficacia académica, presentan menos sentimientos de agotamiento emocional, menores puntuaciones en cinismo, así como una mayor engagement o compromiso con los estudios, caracterizada por vigor, dedicación y absorción. 
Las relaciones entre estos dos constructos pone de manifiesto la influencia positiva de engagement en la eficacia académica del estudiante; apelando a la perspectiva positiva se podría decir que los resultados indican que estudiantes vigorosos y dedicados a sus tareas hacen que se sientan más eficaces en sus estudios y con niveles más bajos de burnout. Según lo expuesto, el fomento del engagement (vigor y dedicación) puede ser una oportunidad para afrontar las tareas académicas y finalizar adecuadamente la formación (eficacia académica) así como para enfrentarse al mundo laboral con mayor probabilidad de éxito.

Se considera necesario realizar más estudios en este tema y un siguiente paso lógico, por la fase incipiente en la que se encuentra el engagement, podría ser profundizar en las relaciones con otras variables en el campo personal (inteligencia emocional, salud, estado afectivo, sentido de coherencia, mecanismos de afrontamiento, etc.) y en la institución universitaria (variables contextuales, organizacionales y académicas), así como ampliar la muestra a otros territorios. Además sería interesante que las Universidades se plantearan planes estratégicos para promocionar la salud en entornos universitarios y potenciar el engagement académico (motivación con los estudios). Estas habilidades son especialmente interesantes para los estudiantes de Enfermería, ya que el compromiso con sus estudios podría implicar mejores resultados académicos y repercutiría posiblemente en un mejor bienestar personal y en unos cuidados de calidad en la población.

\section{REFERENCIAS}

1. Martínez IM, Salanova MS. Niveles de burnout y engagement en estudiantes universitarios: relación con el desempeño y desarrollo profesional. Rev Educ. 2003 [cited 2015 July. 21];(330):361-84. Available from: http://www.wont.uji.es/publicaciones/articulos/item/niveles-de-burnout-y-engagementen-estudiantes-universitarios-relacion-con-el-desempeno-y-desarrollo-profesional 2. Salanova MS, Martínez IM, Bresó E, Llorens GS, Grau GR. Psychological wellbeing among university students: facilitators and obstacles of academic performance. An Psicol. [Internet]. 2005 [cited 2015 July. 21];21(1):170-80. Available from: http://www.um.es/analesps/v21/v21 1/16-21 1.pdf

3. Maslach C, Schaufeli WB Leiter, MP. Job burnout. Annu Rev Ppsychol. 2001;(52):397-422.

Available

from:

http://www.wilmarschaufeli.nl/publications/Schaufeli/154.pdf

4. Maslach C, Jackson SE. Maslach Burnout Inventor. Manual. Palo Alto, Universidad de California: Consulting Psychologist Press; 1981. Available from: http://onlinelibrary.wiley.com/doi/10.1002/job.4030020205/pdf

5. Ramos F. El síndrome del burnout. Madrid: UNED-FUE; 1999.

6. Grisales H, Muñoz Y, Osorio D, Robles E. Síndrome de Burnout en el personal de enfermería de un hospital de referencia lbagué, Colombia, 2014. Enferm. glob. [Internet]. 2016 Ene [cited 2016 Sep 23] ; 15( 41 ): 244-257. Available from: http://scielo.isciii.es/scielo.php?script=sci arttext\&pid=S1695-

61412016001100113\&Ing=es.

7. Tomaschewski-Barlem JG, Lunardi VL, Lunardi GL, Barlem ELD, Silveira RS, Vidal DAS. Burnout syndrome among undergraduate nursing students at a public university. Rev. Latino-Am. Enfermagem [Internet]. 2014 [cited 2015 Oct 19];22(6):934-941. Available from: http://www.scielo.br/scielo.php?script=sci arttext\&pid=S0104$11692014000600934 \& \operatorname{lng}=\mathrm{es}$

8. Guido LA, Goulart CT, Marques da R, Lopes LFD, Ferreira EM. Stress and Burnout among multidisciplinary residents. Rev. Latino-Am. Enfermagem [Internet]. 2012 Dec 
[cited 2015 Oct 21];20(6):1064-1071. Available from: http://www.scielo.br/scielo.php?script=sci arttext\&pid=S0104$1692012000600008 \& \operatorname{lng}=$ en.

9. Salanova M, Schaufeli WB, Llorens S, Peiró JM, Grau RM. Desde el burnout al engagement: ¿una nueva perspectiva? Rev Psicol Trabajo Organ [Internet]. 2000 [cited $2015 \quad$ July. 21];(16):117-134. Available from: http://www.wont.uji.es/publicaciones/articulos/item/desde-el-burnout-al-engagementuna-nueva-perspectiva

10. Seligman MEP, Csikszentmihalyi M. Positive psychology: An introduction. Am Psychol. 2000 [cited 2015 July. 21];(55):5-14. Available from: http://www.ppc.sas.upenn.edu/ppintroarticle.pdf

11. Salanova M, Schaufeli WB. El engagement en el trabajo. Madrid: Alianza Editorial S.A; 2009.

12. Schaufeli WB, Salanova M, González-Romá V, Bakker AB. The measurement of burnout and engagement: A confirmatory factor analytic approach. J Happiness Stud. 2002 [cited 2015 July. 21];3(1):71-92. Available from: http://www.wilmarschaufeli.nl/publications/Schaufeli/178.pdf

13. Uludağ $\mathrm{O}$, Yaratan $\mathrm{H}$. The effect of burnout on engagement: An empirical study on tourism students. J Hosp Leis Sport To. 2010 [cited 2015 July. 21];9(1):13-23. Available from: http://www.sciencedirect.com/science/article/pii/S1877042812011937

14. Salanova M, Bresó E, Schaufeli WB. Towards a spiral model of the efficacy beliefs in the study of burnout and engagement. Anxiety Stress [Internet]. 2005 [cited 2015 July. 21];11(2-3):215-31. Available from: http://www.wilmarschaufeli.nl/publications/Schaufeli/243.pdf

15. Çam Z, Deniz KZ, Kurnaz A. School Burnout: Testing a Structural Equation Model Based on Percieved Social Support, Perfectionism and Stress Variables. Egitim ve bilim-education and science, 2014 [cited 2016 Jun. 20];173(39): 310-325. Available from:

https://apps.webofknowledge.com/full record.do?product=UA\&search mode=General Search\&qid $=1 \&$ SID $=$ N1AxEydR97CcwD47809\&page $=1 \&$ doc $=3$

16. Zhu Y, Liu CC, Guo BM, Zhao L, Lou FL. The impact of emotional intelligence on work engagement of registered nurses: the mediating role of organisational justice. 2015;24(15-16): 2115-2124. DOI: 10.1111/jocn.12807

17. Jing-Ying L, Yan-Hui L, Ji-Peng Y. Impact of learning adaptability and time management disposition on study engagement among chinese baccalaureate nursing students. J Prof Nurs. 2014;30(6):502-10. Available from: http://www.sciencedirect.com/science/article/pii/S875572231400074X

18. Manzano G. Burnout y engagement en un colectivo pre-profesional estudiantes universitarios. Bol Psicol. 2002;74(1):79-102. Available from: http://www.uv.es/seoane/boletin/previos/N74-4.pdf

19. Schaufeli WB, Salanova M. Efficacy or inefficacy, that's the question: Burnout and work engagement, and their relationships with efficacy beliefs. Anxiety Stress Coping. 2007;20(2):177-96. Available from: http://www.wilmarschaufeli.nl/publications/Schaufeli/267.pdf 20. Zhang Y, Gan Y, Cham H. (2007). Perfectionism, academic burnout and engagement among Chinese college students: A structural equation modeling analysis. Pers Indiv Differ. 2007;43(6):1529-1540. Available from: http://www.sciencedirect.com/science/article/pii/S0191886907001602

21. Liébana-Presa C, Fernández-Martínez ME, Ruiz A, Muñoz-Villanueva MC, Vázquez-Casares AM, Rodríguez-Borrego MA. Psychological distress in health sciences college students and its relationship with academic engagement. Rev Esc Enferm USP [Internet]. 2014 [cited 2015 July 21];48(4):715-722. Available from: 
http://www.scielo.br/scielo.php?script=sci arttext\&pid=S0080-

62342014000400715\&lng=en

22. Extremera N, Durán A, Rey L. Emotional intelligence and its relationship with the levels of burnout, engagement and stress in university students. Rev Educ. 2007;(342):239-256. Available

http://www.revistaeducacion.mec.es/re342/re342 12.pdf

23. Upadyayaa K, Salmela-Aroa K. Cross-lagged associations between study and work engagement dimensions during young adulthood. J Posit Psychol. 2014;10(4):346-358.

Available

from:

https://dialnet.unirioja.es/servlet/articulo?codigo $=5055199$

24. Polanco A, Ortiz L, Pérez C, Parra P, Fasce E, Matus O, et. al. Relationship between previous academics background and initial expectations with academic wellbeing of first year of medical students. FEM: Revista de la Fundación Educación Médica. 2014;17(4):205-211. Available from: http://scielo.isciii.es/scielo.php?script=sci arttext\&pid=S2014-98322014000400006

25. García Rodríguez José Juan, Labajos Manzanares Mํㅡ Teresa, Fernández Luque Francisca. Los estudiantes de Grado en Enfermería y su compromiso con los estudios. Enferm. glob. [Internet]. 2015 Abr [citado 2016 Sep 23] ; 14( 38 ): 169177. Disponible en: http://scielo.isciii.es/scielo.php?script=sci_arttext\&pid=S1695$61412015002200009 \&$ Ing $=$ es.

26. Bresó E, Llorens S, Salanova M. Creencias de eficacia académica y engagement en estudiantes universitarios. Jornades de Foment de la Investigació. Castellón: Universitat Jaumel; $2005 . \quad$ Available from: http://repositori.uji.es/xmlui/bitstream/handle/10234/79330/forum 2003 12.pdf?seque nce $=1$

27. Caballero CC, Abelló R, Palacio J. Relación del burnout y el rendimiento académico con la satisfacción con los estudios en estudiantes universitarios. Av Piscol Clin Latinonot. 2007;25(2):98-111. Available from: http://www.redalyc.org/articulo.oa?id=79925207

28. Preciado ML, Vázquez JM. Stress profile and burnout syndrome in mexican dental students of a public university. Rev Chil Neuro-psiquiat [revista en la Internet]. 2010 [cited 2015 July. 21];48(1):11-19. Available from: http://redalyc.uaemex.mx/src/inicio/ArtPdfRed.jsp?iCve $=73013006003$

29. Schaufeli, WB. Bakker, AB. UWES - utrecht work engagement scale: test manual, Department of Psychology, Utrecht University, Utrecht. 2003. Available from: http://www.wilmarschaufeli.nl/publications/Schaufeli/Test\%20Manuals/Test manual U WES Espanol.pdf

30. Schaufeli WB, Martinez IM, Marques-Pinto $A$, et al. Burnout and engagement in university students: A cross-national study. J Cross Cult Psychol 2002; 33(5): 464-481

31. Nunnally JC, Bernstein IH. Psychometric theory. Nueva York: McGraw-Hill; 1994.

ISSN 1695-6141

() COPYRIGHT Servicio de Publicaciones - Universidad de Murcia 